\title{
Jet-cloud Interaction in the Nuclear Region of NGC 1068
}

\author{
J. f. Wang \\ Harvard-Smithsonian Center for Astrophysics, \\ Cambridge, MA 02138 USA \\ email: juwang@cfa.harvard.edu
}

\begin{abstract}
We examine the innermost 100 parsec scale region of the Seyfert 2 galaxy NGC 1068 using a high spatial resolution X-ray image obtained with the Chandra X-ray Observatory, which allows comparison between X-ray emission clumps, optical narrow line ([OIII]) clouds and sub-arcsecond scale radio jet. Based on the combined X-ray, [OIII], and radio continuum morphology, we identify the locations of intense radio jet-cloud interaction. The [OIII] to soft $\mathrm{X}$-ray ratios show that some of these clouds are strongly affected by shock heating. We estimate that the kinematic luminosity of the jet-driven shocks is $6 \times 10^{38} \mathrm{erg} \mathrm{s}^{-1}$.
\end{abstract}

Keywords. Galaxies: active, galaxies: jets, X-rays: ISM

\section{Introduction}

NGC 1068 is the brightest prototype Seyfert 2 active galactic nucleus (AGN), and also one of the closest, with a distance of only $14.4 \mathrm{Mpc}\left(1^{\prime \prime}=72 \mathrm{pc}\right)$. This makes NGC 1068 an optimal target for understanding the feeding and feedback of the supermassive black hole by studying the morphology and kinematics of the different components in the AGN central region. We obtained an unprecedented high spatial resolution X-ray image of the nucleus of NGC 1068 with the Chandra/HRC (ObsID 12705, PI: Fabbiano), where previous ACIS image is affected by pileup.

\section{Results and Implications}

Following Wang et al. (2009) HRC study of NGC 4151, we performed image restoration using the EMC2 algorithm (Karovska et al. 2007). The HRC image of NGC 1068 (Fig. 1a and $\mathrm{b}$ ) has enabled us to compare the sub-arcsecond structures of the X-ray emission and those of the HST NLR clouds, and firmly demonstrates that there is remarkable morphological agreement between the X-ray and the $[\mathrm{OIII}] \lambda 5007$ emission (c.f. Ogle et al. 2003). We further compare the radio images to the X-ray emission (Fig. 1c and d). The newly identified X-ray knot (HST-G) is $0.2^{\prime \prime}$ from the peak of a radio knot, where the collimated radio outflow becomes diffuse. The zoom shows fine details of the spatial relations with the MERLIN $6 \mathrm{~cm}$ features (Gallimore et al. 1996).

The spectral modeling of cloud HST-G revealed the presence of $k T \sim 1 \mathrm{keV}$ hot gas. The kinematics of the ionized gas also suggest jet-cloud impact: the NLR gas at HST-G is strongly kinematically perturbed and shows velocity systems separated by $1500 \mathrm{~km} \mathrm{~s}^{-1}$. The estimated thermal energy content deposited by the jet-ISM interaction is $E_{\text {thermal }}=1.9 \times 10^{53}$ ergs. Taking the velocity of the fast shocks driven by the jet to be $V \simeq 700 \mathrm{~km} \mathrm{~s}^{-1}$, the relevant timescale is the crossing time for the shocks to move through the $1^{\prime \prime}$ (72 pc) extent of the cloud, $t_{\text {cross }}=d / V=10^{5} \mathrm{yr}$. This implies a 

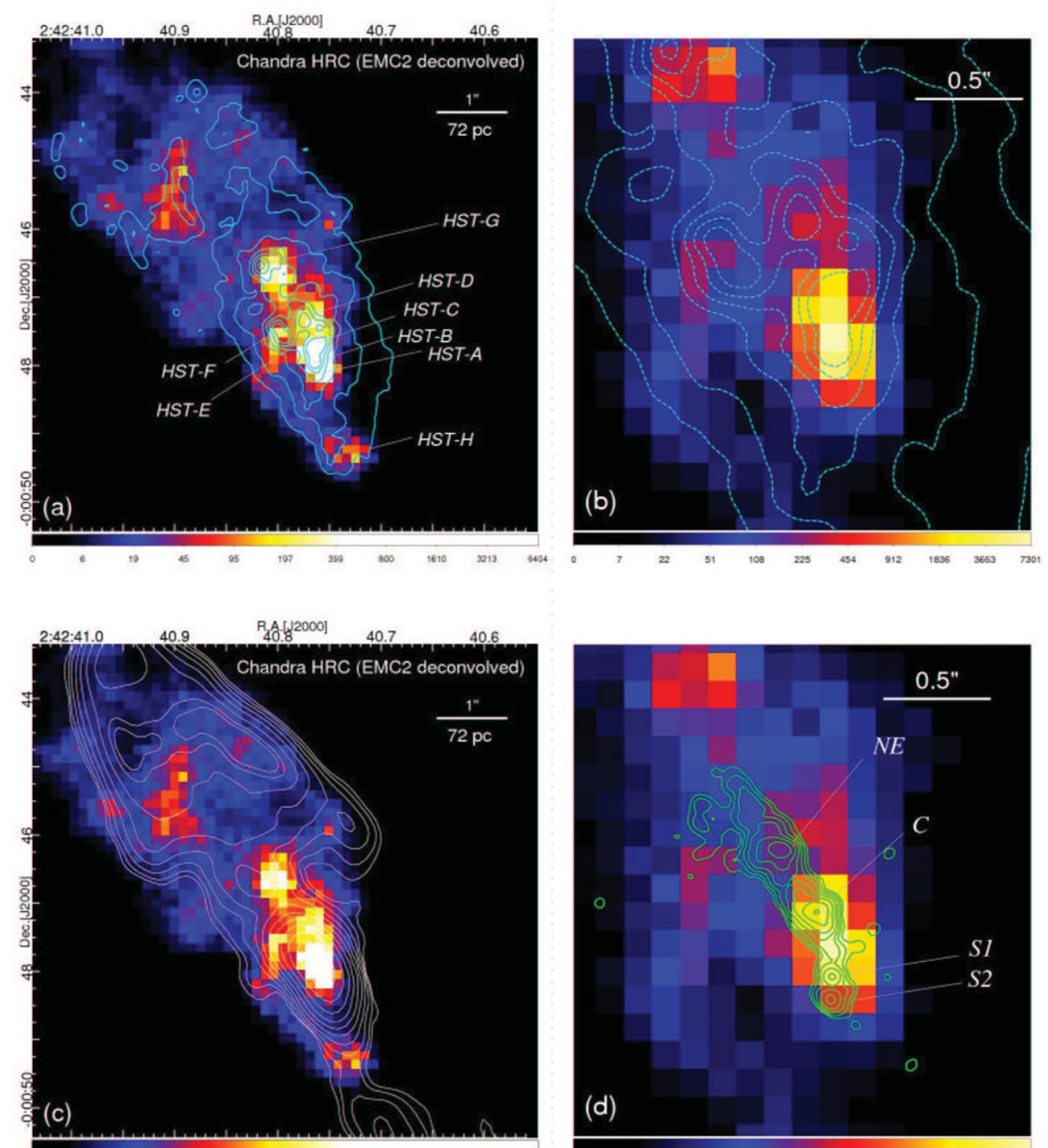

(d)

Figure 1. (a) The deconvolved HRC image of the X-ray emission in NGC 1068 overlaid with the contours of HST/WFPC2 [OIII] $\lambda 5007$ line emission (Capetti et al. 1997). (b) Zoom-in image showing the detailed correspondance of $[\mathrm{OIII}]$ and X-ray features in the innermost region; (c) The deconvolved HRC image overlaid with the contours of VLA $5 \mathrm{GHz}$ emission. (d) The innermost region overlaid with the MERLIN $6 \mathrm{~cm}$ contours.

kinematic luminosity (or energy injection rate) of $L_{K . E .}=6 \times 10^{38} \mathrm{erg} \mathrm{s}^{-1}$, a negligible amount of the jet power $\left(L_{K . E .} / P_{j e t}=10^{-4}\right)$.

\section{References}

Capetti, A., Axon, D. J., \& Macchetto, F. D. 1997, ApJ, 487, 560

Gallimore, J. F., et al. 1996, ApJ, 458, 136

Karovska, M., et al. 2007, ApJ, 661, 1048

Ogle, P. M., et al. 2003, A\&SA, 402, 849

Wang, J., et al. 2009, ApJ, 704, 1195 